\section{Together we are stronger}

Transplantation of neural stem cells (NSCs) is extensively studied as a potential and promising treatment for several neurological disorders. Lee et al. now show that transplantation of NSCs in a mouse model of the neurodegenerative Sandhoff disease can almost double the lifespan of the mice if combined with traditional drugs.

Sandhoff disease manifests itself by the abnormal accumulation of gangliosides within lysosomes throughout the nervous system. This causes mental retardation, motor dysfunction and death in infancy. Lee et al. studied mice lacking $\beta$-hexosaminidase $\left(\mathrm{Hexb}^{-/-}\right)$, an enzyme required for the degradation of gangliosides, as a model for the disease.

The researchers transplanted NSCs into the forebrain and cerebellum of newborn $\mathrm{Hexb}^{-/-}$mice. This extended the lifespan of the mice by $70 \%$, and also preserved their motor function. Although disease onset in mice that had received NSCs was delayed, once they became ill the disease progressed in a pattern similar to that of control $\mathrm{Hexb}^{-/-}$mice. Before onset of the disease, Lee et al. found that the transplanted cells integrated and differentiated normally in the brain, and that they were electrophysiologically indistinguishable from host cells. The effects of prenatal transplantation of NSCs, during active cortical neurogenesis, on extended survival and prolonged motor skills were similar to postnatal transplantation, excluding a simple neuronal replacement model as the predominant mechanism for the prolonged lifespan.

Lee et al. found that NSC-transplanted $\mathrm{Hexb}^{-/-}$mice expressed 5\% of wild-type levels of $\beta$-hexosaminidase, which is sufficient for normal ganglioside metabolism. Consistent with this result, the amount of detectable gangliosides in brain slices was low in NSC-transplanted $\mathrm{Hexb}^{-/-}$ mice, whereas control Hex $b^{-/-}$mice showed a substantial accumulation. Furthermore, in NSC-transplanted $\mathrm{Hexb}^{-/-}$mice, microglial activation - a sign of inflammation recognized as a characteristic of a broad range of neurodegenerative diseases - was greatly reduced.

Substrate reduction therapy, by oral administration of imino sugars, inhibits ganglioside biosynthesis. In combination with NCS-transplantation, imino sugars prolonged the life and motor function of $\mathrm{Hexb}^{-/-}$mice

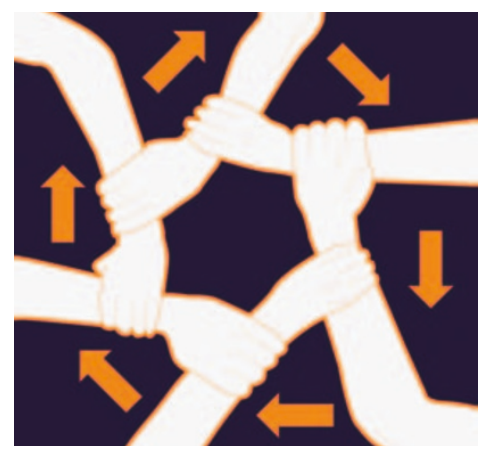

even further, almost doubling the lifespan of some mice. Interestingly, therapeutically functional transplantation could also be achieved with cultured human embryonic NSCs without immunosuppressants.

The pathology of most neurodegenerative diseases is characterized by multiple facets. Hence, as described in this paper, it is most fruitful to view the stem cell not as a unidimensional tool but rather as mediator of a range of actions that each address some of these aspects and which can be effectively combined with other treatments. Therefore, synergistically acting therapies might be the way forward in the treatment of neurodegenerative diseases.

Claudia Wiedemann

ORIGINAL RESEARCH PAPER Lee, J.-P. et al. Stem cells act through multiple mechanisms to benefit mice with neurodegenerative metabolic disease. Nature Med. 13, 439-447 (2007) 